\title{
Performance Evaluation and Analysis of Switching Algorithms in MIMO-OFDM System with Ideal and Non-Ideal CSI
}

\author{
Yosra Mlayeh, Fethi Tlili, Fatma Rouissi, Ilham Ouachani, Adel Ghazel \\ CITRA'COM Research Laboratory, Engineering School of Communications (SUP'COM), Tunis, Tunisia \\ E-mail: fethi.tlili@supcom.rnu.tn
}

Received September 12, 2010; revised October 15, 2010; accepted November 17, 2010

\begin{abstract}
In this paper we analyzed the bit error rate performance of a switching algorithm between spatial multiplexing and diversity for an OFDM MIMO system with ideal channel state information. The effect of channel estimation error was studied and we verified by simulations that the spatial multiplexing outperforms the switching algorithm. Given that the switching algorithm is based on the comparison of the channel matrix Demmel condition number to a threshold, its accuracy is compromised when channel estimation error increases. As a first intuitive solution, we proceeded to the adaptation of the threshold, but this didn't lead to a pertinent improvement for the main reason that channel estimation errors did affect the MIMO techniques which use different constellation. Based on that, we proposed a new estimation technique that improved the bit error rate performance significantly.
\end{abstract}

Keywords: MIMO Diversity, Spatial Multiplexing, OFDM, Demmel Condition Number, Switching Algorithm, Channel Estimation

\section{Introduction}

MIMO systems, with the integration of the spatial dimension, represent a good solution to improve the rate and the robustness of transmission systems without increasing the bandwidth of the system [1]. In addition, using OFDM with MIMO allows simplifying the equalization at the receiver [2]. MIMO-OFDM techniques were introduced by the IEEE $802.16 \mathrm{e}-2005$ specifications as a solution to improve the quality of service [3].

MIMO diversity (MD) and Spatial Multiplexing (SM) are the two most known techniques used in MIMO channels. Diversity techniques use two or more antennas in the transmitter and the receiver side to improve the wireless link quality and are not designed to increase the peak data rate of the system [4]. An effective and simple transmit diversity scheme namely Space Time Block Coding (STBC) is proposed by Alamouti [5]. It encodes the signal through two transmit antennas and in time to enhance significantly the BER while preserving a unit code rate. STBC has been generalized for more than two antennas in [6].

In addition, spatial multiplexing techniques offer higher peak throughput by transmitting independently and separately encoded data signals from each of the multiple transmit antennas $[7,8]$.

Furthermore, when the MIMO technique is selected based on feedback information about the channel state, this can lead to higher link robustness. This aspect involves studying appropriate adaptation algorithms allowing the selection of the most appropriate MIMO technique for given channel conditions. The transmitter blocs' specifications such as the MIMO technique and the modulation scheme are adjusted according to the current state of the wireless mobile channel instead of being designed based on the worst case scenario. This approach provides a much more efficient use of the available resources and gives a practical cognitive radio strategy.

This is especially true for the case of a MIMO-OFDM system since it is very flexible and there are a great number of parameters which can be adapted as the usual modulation, the coding scheme and the MIMO scheme.

Several Adaptation Algorithms are proposed to enhance system performances in terms of Bit Error Rate (BER) [9-12]. Other algorithms are proposed to maxi- 
mize capacity gains [13-17].

To enhance system performances, the MIMO switching technique was discussed for a fixed data rate in case of narrowband MIMO channels [9-11]. Either multiplexing or diversity was chosen based on the instantaneous channel state and the decision is conveyed back to the transmitter via a low-rate feedback channel. The Demmel condition number of the instantaneous channel matrix was proposed as a parameter based on the minimum Euclidean distance of constellations in order to characterize the suitability of a given instantaneous channel matrix for SM compared to MIMO Diversity. In [12], the authors have studied the suitability of a complex $2 \times 2$ MIMO-OFDM channel matrix for SM using instantaneous measurements of its channel Demmel condition number in several indoor environments.

Other works are interested to enhance the capacity gains using adaptation algorithms, where the selection criterion is based on the knowledge of the Signal to Noise Ratio (SNR) and the determinant of MIMO channel matrix $[13,14]$. These works focus primarily on the case of delay insensitive applications for which throughput maximization is the criterion for adaptation, and examine performances of the adaptive MIMO-OFDM system in a realistic outdoor environment, modeled using ray tracing software.

In [15], it was shown that at the same spectral efficiency, Alamouti's STC combined with maximum-ratio combining at the receiver significantly outperforms the $2 \times 2$ spatial multiplexing scheme at high values of the signal-to noise ratio (SNR). In this work, the selection of the MIMO option is included in link adaptation to maximize network capacity, and operating SNR regions are determined for different modulation, coding and MIMO combinations.

Moreover, the impact of mutual antenna coupling on the ergodic capacities of statistical beamforming and spatial multiplexing transmission strategies is analyzed $[16,17]$. Adaptive switching between combinations of transmission strategies and antenna array configurations (using reconfigurable antenna arrays) is shown to produce maximum capacity gains.

Hence, we deduce that the approach of physical layer adaptation is becoming more and more an interesting subject in the telecommunication area. Nevertheless, previous works assumes ideal Channel State Information (CSI) and no feedback delay.

So, to evaluate the performances of theses algorithms while considering practical contexts, this paper considers the effect of channel estimation errors on a $2 \times 2$ MIMO OFDM switching algorithm.

To summarize, using the Demmel condition number as a selection metric, we:
1) Evaluate performances of OFDM switching algorithm in ideal CSI.

2) Study the effect of channel estimation on switching algorithm performances.

3) Propose three methods to improve performances of this switching algorithm in practical contexts with non ideal CSI.

This paper is organized as follows: the second section describes the OFDM-MIMO based system following with switching algorithm. The third section deals with MIMO-OFDM channel estimation effect on the switching technique. After a brief review of estimation methods, performances of the switching algorithm with non ideal CSI are showed and analyzed. Then, two solutions are proposed to improve these performances. The first one, although its additional complexity, reaches the performances of ideal CSI case. The second one enhances considerably performances of switching algorithm without any change in the receiver design. In Section 4, Conclusion and perspectives of the work are outlined.

\section{MIMO-OFDM System with Switching Algorithm}

\subsection{System Model}

In this work, we consider The MIMO-OFDM transceiver following WiMAX standard as depicted in Figure 1. Different blocs are defined following 802.16e -2005 specifications [3].

In the transmitter side, data are encoded using convolutional codes. After bitwise interleaving, bits are mapped to M-QAM symbols. Then, the MIMO encoder is fed and OFDM samples are computed via the IFFT. Finally, the cyclic prefix is added.

The MIMO module can be used to spatial Multiplexing, Spatial Diversity or beamforming. In this paper, a switching algorithm is used to select between Spatial Multiplexing and MIMO Diversity in order to enhance performances of the MIMO system in terms of BER. To compare performances offered by proposed algorithms to that proposed in the literature, we adopt $2 \times 2$ MIMO architecture, which means that we use two antennas in the transmitter side $\left(N_{t}=2\right)$ and two antennas $\left(N_{r}=2\right)$ in the receiver one.

SM Techniques are known to increase the throughput at a given SNR. The base station transmits independent data streams from each transmit antenna to the subscriber's stations. It could be implemented using Zero-forcing (ZF), Maximum likelihood or MMSE (Minimum Mean Square Error) detectors [8]. The ZF detector inverts the channel matrix to detect the transmitted symbols. Even though it suffers from poor performances at low SNR, it 


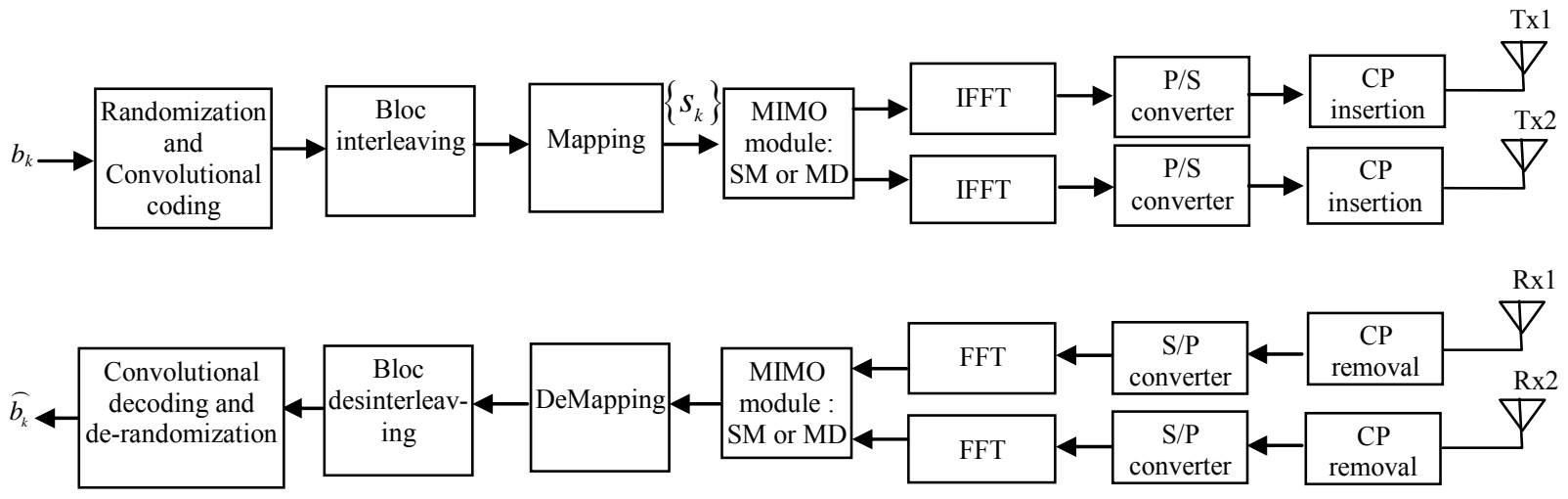

Figure 1. Block diagram of the MIMO-OFDM transceiver.

is employed in this work as the detection scheme because it has a very small complexity and does not depend on the modulation type.

MD uses two or more antennas in the transmitter and the receiver side to improve the wireless link quality. The main idea behind antenna diversity techniques is to produce different replicas of the transmitted signal to the receiver $[5,6]$. These replicas are sent over the propagation channel. Due to this redundancy, the receiver can decode the transmitted signal even in fading conditions, as long as they all do not fade simultaneously. In this work, we use the Alamouti code as a Space Time Block Code (STBC).

\subsection{Switching Between SM and MD}

Link adaptation plays a central role in regulating the use of radio resources. The idea behind switching algorithm is to dynamically adapt the MIMO technique and the modulation scheme to channel conditions in order to achieve the highest Bit Error Rate (BER) performances. Several channel metrics were proposed as selection criteria. The well-known metrics are Signal to Noise ratio, channel matrix determinant $[13,14]$, the minimum Euclidean Distance $[9,10]$ and the Demmel condition number $[11,12]$. In this work, the OFDM switching between the $\mathrm{SM}$ and the DM techniques is based on the Demmel condition number $\left(K_{d}\right)$ criterion. It can provide information about the invertibility of the channel which provides knowledge about its suitability for use in either SM or MD operational modes [18]. The Demmel condition number for a random real or complex matrix $\boldsymbol{H}$ is defined in [19] as:

$$
K_{d}\left(\boldsymbol{H}_{n}\right)=\frac{\left\|\boldsymbol{H}_{n}\right\|_{F}}{\lambda_{\text {min }}\left(\boldsymbol{H}_{n}\right)}
$$

where $\boldsymbol{H}_{n}$ denotes the MIMO channel matrix of the $n^{\text {th }}$ subcarrier, $\lambda_{\min }\left(\boldsymbol{H}_{n}\right)$ refers to the minimum singular value of $\boldsymbol{H}_{n}$ and \|\|$_{F}$ refers to the Frobinüs norm.

The Demmel condition number measures how ill posed a given matrix is. Physically, in [7] it was shown that this metric provides a comparison between the minimum signal constellation distance needed to support SM and MD modes of operation for a given channel.

Considering the expression of the Demmel condition number given by Equation (1), one sufficient condition that multiplexing will be better than diversity for a given channel matrix is given in [9] by:

$$
K_{d}\left(\boldsymbol{H}_{n}\right) \leq \frac{d_{\min , S M t}}{d_{\min , M D t}},
$$

where $d_{\text {min,SMt }}$ and $d_{\text {min,MDt }}$ are minimum Euclidean distances at the transmitter side in case of SM and MD, respectively.

The switching algorithm adds an extra processing in either the transmitter or the receiver. The decision is first computed by the receiver, for every subcarrier, then, it is sent back to the transmitter via a low rate feedback channel. Depending on the received decision vector, the transmitter will switch between the two MIMO techniques.

To ensure that an overall rate of $R$ bits per codeword is maintained, symbols are derived from a constellation with $R$ bits per symbol when MD technique is chosen and with $R / N T$ bits per symbol when SM is chosen.

Given that every subcarrier has its appropriate channel matrix and consequently its Demmel condition number, the decision is taken for every subcarrier. The switching algorithm will select the adequate MIMO technique in every subcarrier.

\subsection{Performance Evaluation with Ideal CSI}

Performances in terms of BER of Demmel condition number was evaluated in case of narrowband channels in [9]. In order to evaluate these performances of switching 
algorithm, in case of broadband channels, computer simulations are carried out using the same physical layer as specified in 802.16e standard and summarized in Table 1. The multipath channel is modeled following the Stanford University Interim (SUI4) channel model [20, 21].

In each channel realization, we assume having a time invariant Rayleigh fading channel for every block of three OFDM periods. Simulations are done such that Bit Error Rates (BER) is averaged for every 300 channel realizations.

In Figure 2, we consider the subcarrier based switching algorithm (denoted as "switching") in comparison with SM and MD performances. We also illustrate performances of the "keep method" as referred in literature, which applies the switching algorithm for clusters of subcarriers. In fact, the choice of multiplexing or diversity could be made for a group of subcarriers whose total band corresponds to the coherence bandwidth of the channel, and this reduces the feedback information.

If we use for example the SUI4 channel model (the delay spread values of up to $4 \mu \mathrm{s}$ ), the multipath fading can be considered as flat fading over $50 \mathrm{KHz}$ frequency width. So, as the sub-band width $B s$ of an OFDM mobile WiMAX system is equal to $10.94 \mathrm{KHz}$, the cluster would be composed of 5 subcarriers and the feedback would be reduced by a factor of 5 .

First, we notice that results show a gain of $2 \mathrm{~dB}$ at $\mathrm{BER}=10^{-3}$. This gain outlined by [9] is of about $1 \mathrm{~dB}$ at higher SNRs and a marginal gain at lower SNRs.

Also, the Figure 3 shows that using the "keep method" gives same performances as the switching applied in every subcarrier, with a reduced feedback.

\section{Analysis and Improvement of Switching Algorithm Performances with Non-Ideal CSI}

In the previous part of this work, the switching algorithm has been developed and studied under the hypothesis of perfect channel Knowledge. Nevertheless, in practical context, channel estimation is a mandatory task allowing the receiver to do the Frequency or Temporal Equalization. Hence, when the channel is estimated, estimation errors may induce erroneous decisions that affect switching algorithm performances.

In this section, we propose to study the effect of channel estimation on the switching algorithm. After a brief review of channel estimation techniques, we evaluate and analyze performances of the switching method in case of imperfect channel knowledge. Then, two solutions are presented to improve these performances.
Table 1. Simulations settings.

\begin{tabular}{lc}
\hline Channel bandwidth & $5 \mathrm{MHz}$ \\
Sub carrier spacing & $\Delta_{f}=10.94 \mathrm{KHz}$ \\
OFDM symbol period & $T_{s}=9.14 \mu \mathrm{s}$ \\
Cyclic Prefix & $T_{g}=\frac{T_{s}}{4}=22.8 \mu \mathrm{s}$ \\
Number of sub carriers & $N_{F F T}=512$ \\
Modulation scheme & 16-QAM for MD and 4-QAM for SM \\
Power delay profile & SUI4 channel model \\
Sampling Frequency & $F_{e}=\Delta_{f} * N_{F F T}=5.6 \mathrm{MHz}$ \\
\hline
\end{tabular}

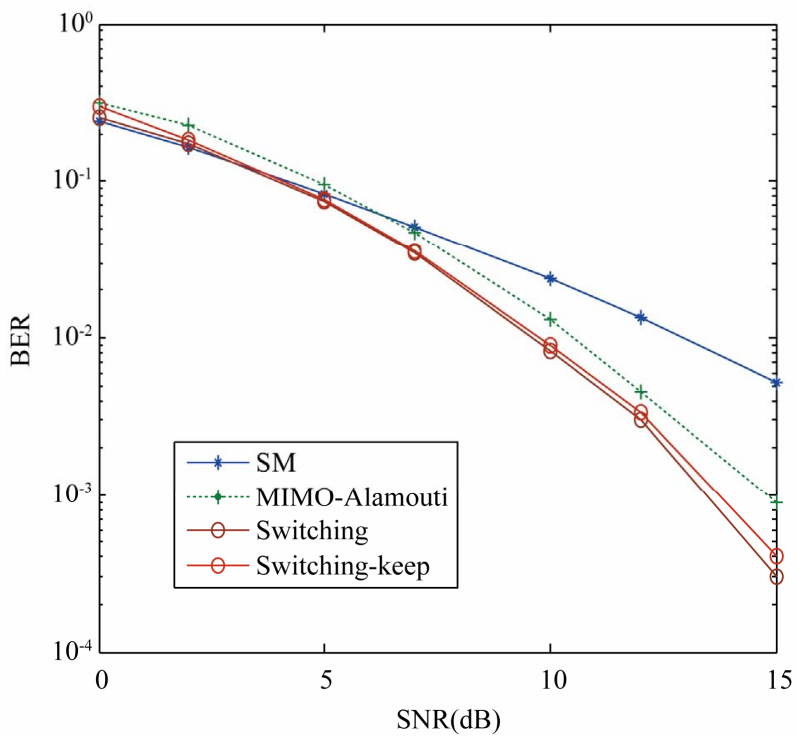

Figure 2. Performances of Subcarrier based switching algorithm.

\subsection{Review of Channel Estimation Techniques in MIMO-OFDM Context}

Channel estimation is needed in multipath channels in order to perform frequency domain equalization. In MIMO-OFDM context, we have a $\left(N_{r} x N_{t}\right)$ channel matrix, so MIMO channel estimation is equivalent to estimate $\left(N_{r} x N_{t}\right)$ SISO channels [22,23], and specific arrangements and pilot positions must be respected at the transmitter by taking into account the number of transmit antennas. The channel estimation is performed in two steps: the channel detection at pilot tones, then interpolation to unknown pilots.

In this work, we use the com-type pilot channel estimation, which is suitable for fast variable channel [24]. In this technique, pilot tones are inserted in specific subcarriers of each OFDM symbol, and the interpolation is 


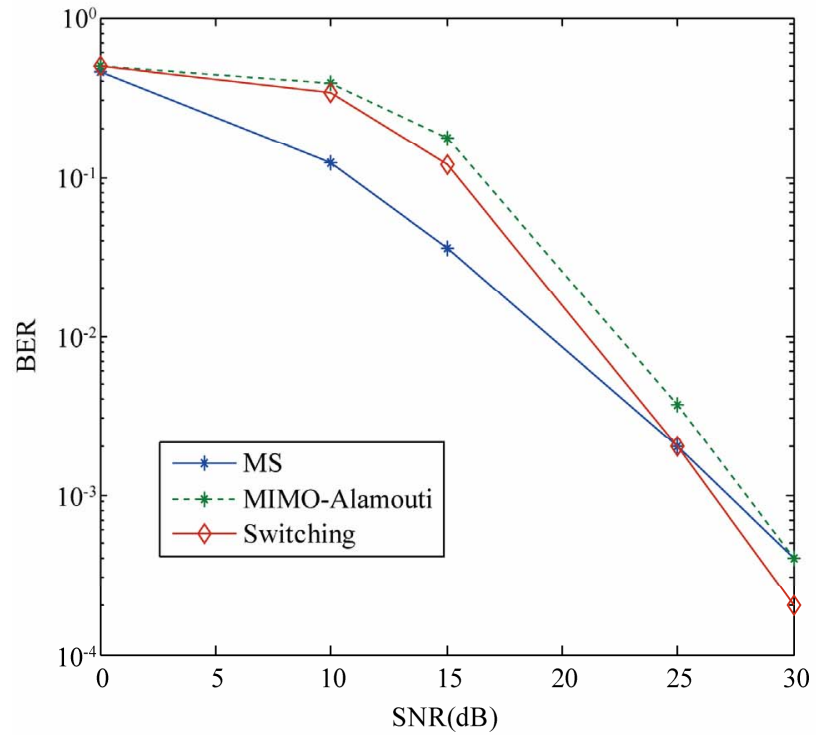

(a)

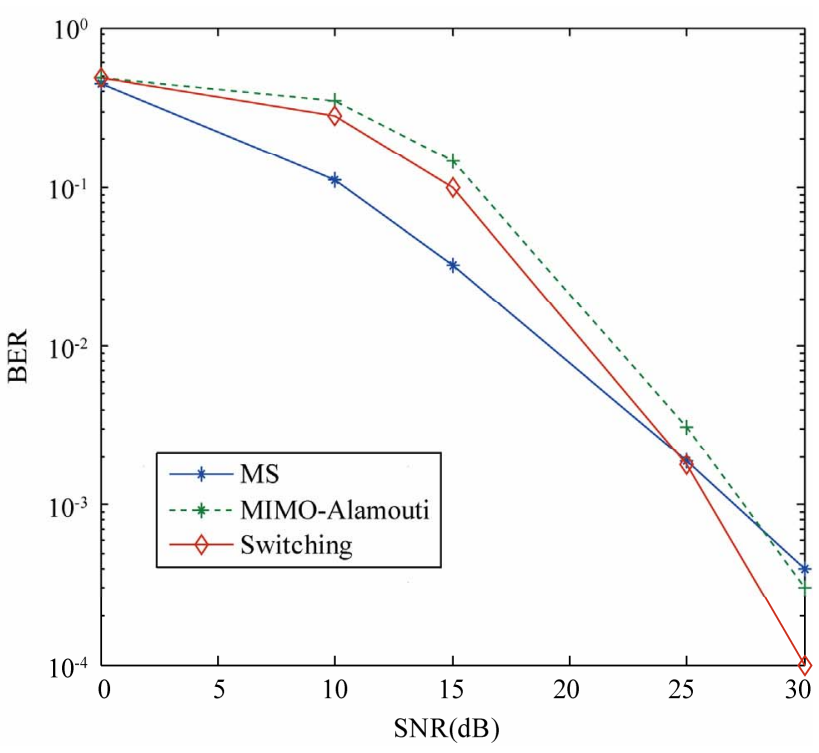

(b)

Figure 3. Performances of switching algorithm in case of estimated channels. (a) LS channel estimation; (b) MMSE channel estimation.

needed to estimate the channel coefficients in data subcarriers.

\subsection{Performance Evaluation of the Switching Algorithm with Non-Ideal CSI}

The objective is to show, by simulation, the effect of the channel estimation on the switching algorithm. We adopt same simulation settings as those described in Subsection 2.3 .

We illustrate in Figure 3 switching performances, in terms of BER using LS and MMSE estimators.

Figure 3 shows that whatever is the channel estimation technique, the switching algorithm doesn't work properly. Also, we deduce that the spatial multiplexing is the best to apply when the channel is estimated, and the MIMO diversity is the most affected. This is due to the constellation size used in each MIMO technique. In fact, the SM scheme is associated to a 4-QAM modulation; however the MD scheme works with 16-QAM modulation, which is more affected by estimation errors.

To explain the performances degradation of the switching, we analyze the influence of channel estimation errors on the $K_{d}\left(\boldsymbol{H}_{n}\right)$ computation, which make decisions of the switching algorithm erroneous. Let's denote $\Delta k d_{n}$ the Demmel condition number variation, as defined in (3).

$$
\Delta k d_{n}=K_{d}\left(\widehat{\boldsymbol{H}}_{n}\right)-K_{d}\left(\boldsymbol{H}_{n}\right) .
$$

where $\widehat{\boldsymbol{H}}_{n}$ denotes the estimated channel matrix.

The decision threshold of the switching algorithm is given by the term $\xi=\frac{d_{\min , S M t}}{d_{\min , M D t}}$. When the receiver computes the Demmel condition number of the estimated channel matrix $K_{d}\left(\widehat{\boldsymbol{H}}_{n}\right)$, a decision error may occur in two cases, the first is when $K_{d}\left(\widehat{\boldsymbol{H}}_{n}\right)>\xi$ wheras $K_{d}\left(\boldsymbol{H}_{n}\right)<\xi$, and the second is when $K_{d}\left(\widehat{\boldsymbol{H}}_{n}\right)<\xi$ wheras $K_{d}\left(\boldsymbol{H}_{n}\right)>\xi$.

According to Figure 3, as the SM is the more suitable MIMO technique, the switching algorithm had to choose it, which is not the case. So the probability of SM selection is reduced when the channel is estimated.

To verify this deduction, we study this probability, equal to $\operatorname{Pr}\left(K_{d}\left(\boldsymbol{H}_{n}\right) \leq \xi\right)$. For that, we use the probability distribution of $K_{d}\left(\boldsymbol{H}_{n}\right)$ which is defined in $[25,26]$, for $m \times m$ square matrix with Independent and Identically Distributed elements, by the following equation:

$$
\operatorname{Pr}\left(K_{d}\left(\boldsymbol{H}_{n}\right) \leq \xi\right)=\left(1-m / \xi^{2}\right)^{m^{2}-1}, \xi>\sqrt{m}
$$

As well, the probability distribution of $K_{d}\left(\widehat{\boldsymbol{H}}_{n}\right)$ is given by (5):

$$
\begin{aligned}
\operatorname{Pr}\left(K_{d}\left(\widehat{\boldsymbol{H}}_{n}\right) \leq \xi\right) & =\operatorname{Pr}\left(K_{d}\left(\boldsymbol{H}_{n}\right) \leq \xi-\Delta k d_{n}\right) \\
& =\left(1-m /\left(\xi-\Delta k d_{n}\right)^{2}\right)^{m^{2}-1}
\end{aligned}
$$

with $\xi>\left(\sqrt{m}+\Delta k d_{n}\right)$.

Curves in Figure 4 give the CDF (cumulative distribution function) of $K_{d}\left(\boldsymbol{H}_{n}\right)$. We also verify the theoretical expression of $K_{d}\left(\widehat{\boldsymbol{H}}_{n}\right)$ with simulations. 


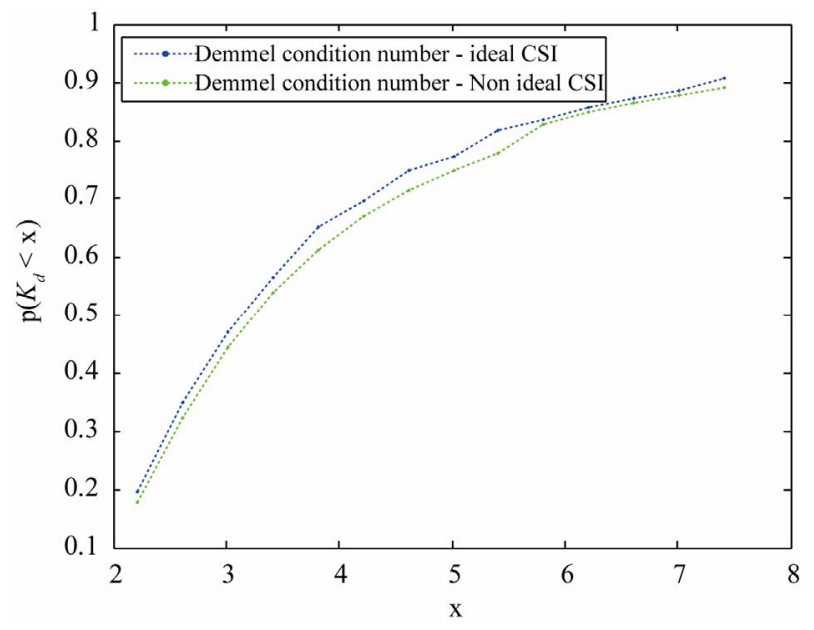

Figure 4. CDF of $K_{d}$ in cases of ideal and non ideal CSI.

As mentioned above, the probability of choosing the SM technique decreases, and makes decision errors occurs when applying the switching algorithm.

Also, when analyzing the Expression (5), we deduce that the Demmel condition number variation $\left(\Delta k d_{n}\right)$ could be expressed by a shift in the decision threshold $\left(\xi-\Delta k d_{n}\right)$. Hence, one idea consists of using an "optimal threshold" to offset the effect of channel estimation on the decision metric. This is the topic of the next subsection.

\subsection{Analysis of Erroneous Switching Decision-Optimal Threshold Technique Description}

The objective is to settle, for each estimator NMSE (Normalized Mean Square Error) value, an optimal threshold $\xi_{\text {opt }}$ that minimizes the switching decision errors by compensating $\Delta k d_{n}$. The MSE is defined in (6), for $N_{s}$ channel realizations.

$$
N M S E=\frac{1}{N_{s}} \sum_{k=1}^{N_{s}} \frac{\left\|\boldsymbol{H}_{k}-\widehat{\boldsymbol{H}}_{k}\right\|^{2}}{\left\|\boldsymbol{H}_{k}\right\|^{2}}
$$

for that, we apply the switching algorithm to estimate channels with different NMSE values, and we determine, for each of them, the optimal threshold value that allows the same switching decision as the one deduced in the case of a perfect channel knowledge. The $N_{s}$ value is chosen equal to 800 , for which a further increase holds negligible impact on $\xi_{\text {opt }}$. We illustrate in Figure 5 resulted optimal thresholds as functions of NMSE.

The curve of Figure 5 shows that $\xi_{\text {opt }}$ increases with NMSE, which means that probability of SM selection should increase as much as the estimation error is important. This agrees with results in Figure 4 where we show that the variation $\Delta k d_{n}$ take positive values and according to (5), the SM selection probability decrease as the $\Delta k d_{n}$ increase.

Basing on several previous works that are interested to study the relation between SNR and MSE in estimated channels [27], the optimal threshold technique is summarized according to the following steps:

1) Fix the SNR value which is considered as a metric of Channel State Information.

2) Determine, from the SNR value and its corresponding MSE, $\xi_{\text {opt }}$ value that will be used in the switching algorithm.

3) Apply the switching algorithm, using $\xi_{\text {opt }}$, to choose the appropriate MIMO technique.

To validate this extra-processing, percentages of switching decision errors, when using $\xi$ are compared to those when using $\xi_{o p t}$. As shown in curves of Figure 6,

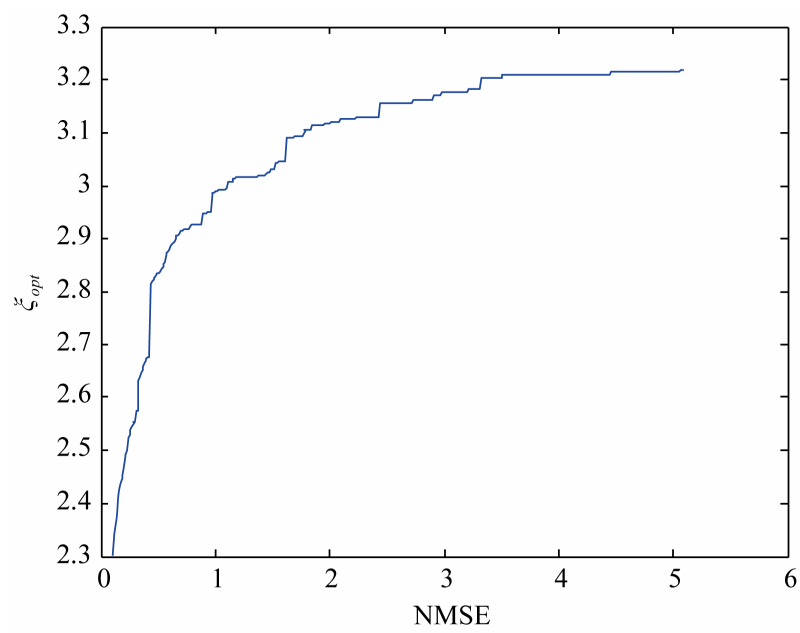

Figure 5. Optimal threshold fixing for different values of NMSE.

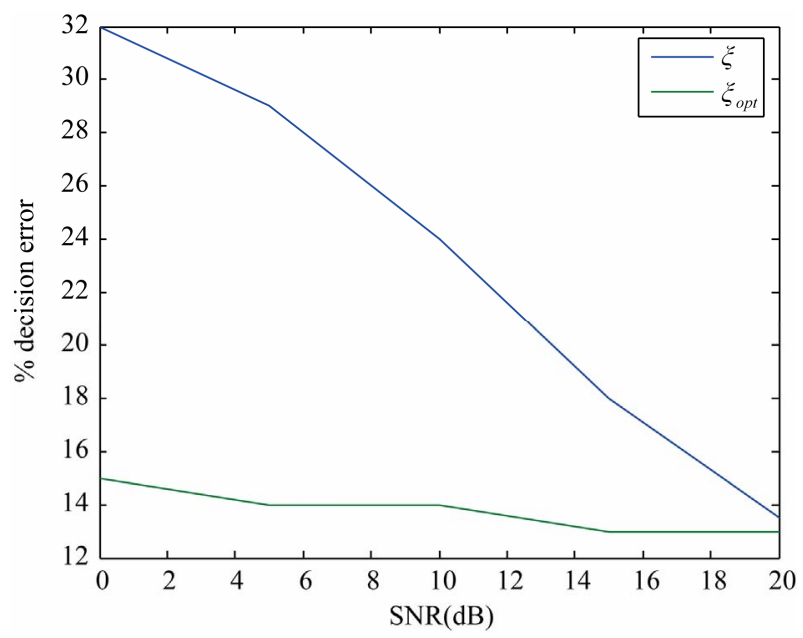

Figure 6. Percentage of erroneous decisions - Comparison between using $\xi$ and $\xi_{\text {opt }}$. 
using optimal threshold improve considerably the switching decision.

To study the impact of the decision on performances of switching module in terms of BER, we evaluate performances of switching algorithm with threshold adaptation compared to those using fixed threshold.

The curves of Figure 7 show that the amelioration added by adaptation process is poor and the switching technique performances are no longer degraded compared to Spatial Multiplexing. So, we conclude that channel estimation errors will not create decision errors only and that the switching algorithm degradation is also due to the effect of these estimation errors on two MIMO modes at the time of equalization. This is due to the dependence of channel estimation error on M-QAM constellation in Rayleigh fading channels [28,29]. In fact, estimation errors are as more important as the constellation is greater. The modulation order used for every technique (4-QAM and 16-QAM) exhibits an error floor for low values of SNR $(<25 \mathrm{~dB})$ and this has an impact on performances of switching algorithm. The blue curves show that in SNR range [0-25], the SM technique gives better performances than switching algorithm. Nevertheless, for high values of SNR, The switching algorithm works properly. Hence, developing a robust channel estimation method is essential to exploit performances offered by the advanced MIMO algorithms as the switching algorithm.

\subsection{Proposal Solutions to Improve the Switching Algorithm with Non-Ideal CSI}

In this subsection, we describe two solutions to reduce

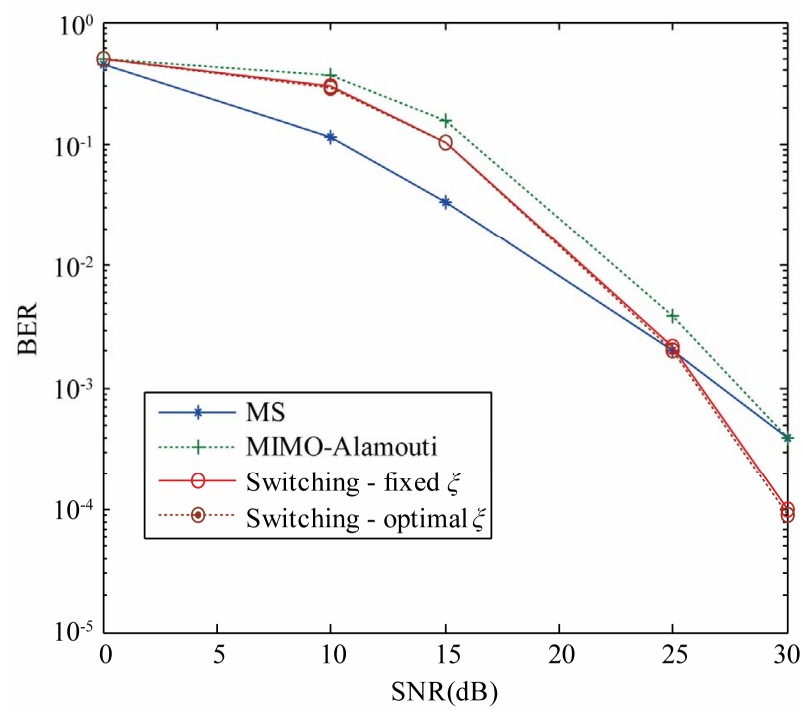

Figure 7. Performances of the switching algorithm using $\xi_{\text {opt }}$ in case of estimated channels. estimation errors in order to improve the switching algorithm performances even in low SNR. The first technique is to develop a robust method of channel estimation; the second is to use a reduced constellation in the estimation by describing a novel channel estimation process.

\subsubsection{Robust Channel Estimation}

In [30], we have developed an IFFT/FFT LS estimation channel method based only on the knowledge of the power delay profile of the channel. It consists of adding an extra IFFT/FFT processing to better estimate channel coefficients. This method is summarized according to the following steps [30]:

- Apply the conventional LS estimator to determine a first approximation of channel frequency response coefficients.

- Convert LS estimated coefficients into the time domain, using the IFFT transform. The result of this step is impulse response coefficients $\hat{h}_{n}$ for $n=$ $0, \cdots,(N-1)$ where $N$ is the subcarrier number.

- Exploit the information providing by the path delay which is the number $L$ and positions of taps. So, corresponding impulse response coefficients are kept, the remaining $(N-L)$ non considered coefficients will be set to zero. This is conveyed by (6).

$$
\tilde{h}_{n}=\left\{\begin{array}{cc}
\hat{h}_{n} & \text { if } n \text { in }\left\{l_{0}, l_{1}, \cdots, l_{L-1}\right\} \\
0 & \text { else }
\end{array}\right.
$$

where $\left\{l_{0}, l_{1}, \cdots, l_{L-1}\right\}$ is the set of taps index.

- Apply the FFT transform to the new impulse response $\tilde{h}$ to obtain frequency response coefficients $\tilde{H}_{n}$ for $n=0,1, \cdots, N-1$.

\subsubsection{New Switching Process}

In Subsection 3.3, we've shown that the switching algorithm performances degrade because of different constellation size used by the two MIMO modes. So, the idea of this part of the work consists of using a small constellation size to improve the channel estimation and so the switching algorithm.

For that, we propose to apply a training process based on the transmission of two OFDM symbols, during one OFDM symbol period, using the 4-QAM modulation with $\mathrm{SM}$ as a MIMO scheme. By assuming that the channel is constant over three OFDM symbol periods, the information provided after this training period is exploited to:

- Compute the Demmel condition number to have knowledge about the "goodness" of every subcarrier for use in SM or MD. The decision vector will be applied to do the switching in the next two OFDM symbol periods.

- Apply the estimated channel coefficients in equa- 
lization task along three OFDM symbol periods.

Thus, in the first OFDM symbol period, we require the $\mathrm{SM}$ as a MIMO technique, and in the remaining two OFDM symbol periods, the switching algorithm is applied with a good decision vector and good channel estimation.

\subsubsection{Simulation Results}

First, Figure 8 shows BER performances of MIMO system using the improved estimation as described in Subsection 3.4.1.

Figure 8 shows that the improved estimation offers BER performances that are close to the perfect case with good channel knowledge, for each of the three techniques (SM, MD and the switching). Also, the switching algorithm works properly and performs better than MIMO Diversity and spatial multiplexing. This will keep its performances and advantages.

Then, Second results, given in Figure 9, deal with new switching process validity, we present, BER performances, versus SNR.

As expected, Figure 9 shows that the new estimation process enhances the performances of switching algorithm compared to SM and MD techniques. Results show a gain of about $5 \mathrm{~dB}$ at $\mathrm{BER}=10^{-3}$.

\section{Conclusions}

In this paper, we presented an OFDM switching algorithm adapted to MIMO-OFDM systems. The switching whose criterion is based on the value of Demmel Condition number was done between Spatial Multiplexing and MIMO diversity. Simulations results show that better performances are offered by the switching algorithm in case of perfect channel knowledge. A gain of $2 \mathrm{~dB}$ is outlined at a BER $=10^{-3}$. However, these performances are damaged when we consider a practical context with non-ideal CSI. Especially, the MIMO Diversity is more affected by estimation errors than the Spatial Multiplexing. This is due to the size of the used constellation in each technique. In fact, in MIMO diversity, we employ a greater constellation (16-QAM) than that used in the SM technique (4-QAM).

For that, two solutions are proposed to improve performances of the switching algorithm under these conditions. The first consists of using a robust channel estimation technique by adding an extra-processing to the LS estimator. The second solution is to employ a training-processing with 4-QAM constellation in order to fix the decision vector to be used in the switching, and at the same time to make better channel estimation with a reduced constellation size. Simulation results of both solu-

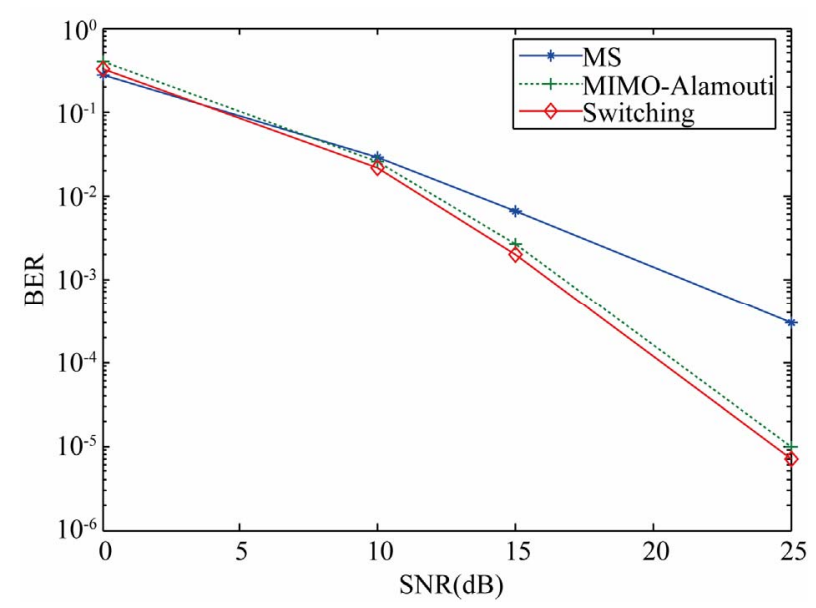

Figure 8. Performances of subcarrier based switching algorithm with improved channel estimation.

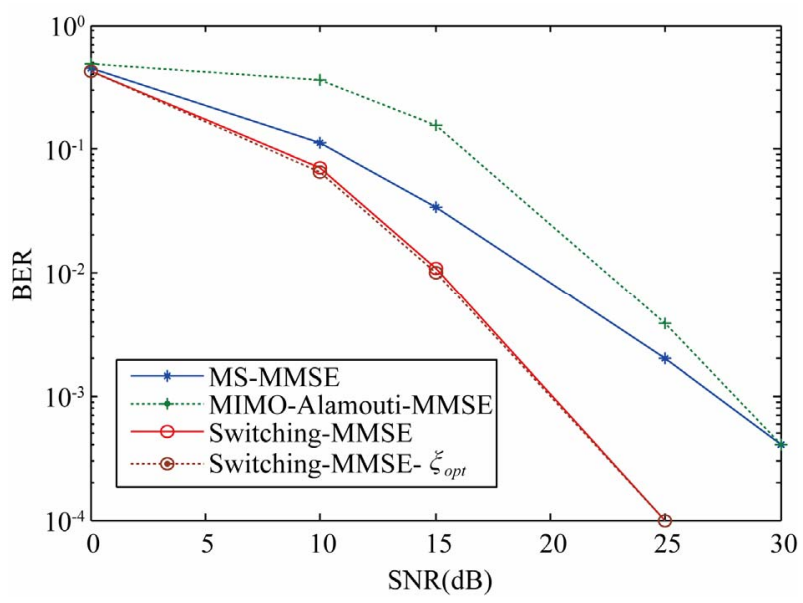

Figure 9. Performances of subcarrier based switching algorithm with new estimation process.

tions showed performances close to those offered under ideal-CSI condition.

\section{References}

[1] H. Sampath, S. Talwar, J. Tellado, et al., "A FourthGeneration MIMO-OFDM Broadband Wireless System: Design, Performance, and Field Trial Results," IEEE Communications Magazine, Vol. 40, No. 9, September 2002, pp. 143-149.

[2] M. D. Batariere, J. F. Kepler, T. P. Krauss, et al., "An Experimental OFDM System for broadband Mobile Communications," Proceedings of IEEE VTS 54th Vehicular Technology Conference, Atlantic City, Vol. 4, 7-11 October 2001, pp. 1947-1951.

[3] IEEE Standard 802.16e, "IEEE Standard for Local and Metropoltain Area Networks, Part 16: Air Interface for Fixed and Mobile Broadband Wireless Access Systems, Amendment 2: Physical and Medium Access Control 
Layers for Combined Fixed and Mobile Operation in Licensed Bands and Corrigendum 1," February 2006.

[4] D. Gesbert, M. Shafi, D. Shiu, P. J. Smith and A. Naguib, "From Theory to Practice: An Overview of MIMO Space Time Coded Wireless Systems," IEEE Journal on Selected Areas in Communications, Vol. 21, No. 3, April 2003, pp. 281-302.

[5] S. M. Alamouti, "A Simple Transmit Diversity Technique for Wireless Communications," IEEE Journal on Selected Areas in Communications, Vol. 16, No. 8, October 1998, pp. 1451-1458.

[6] V. Tarokh, H. Jafarkhani and A. R. Calderbank, "Space Time Block Codes from Orthogonal Designs," IEEE Transactions on Information Theory, Vol. 45, No. 5, July 1999, pp. 1456-1467.

[7] A. J. Paulraj and T. Kailath, "Increasing Capacity in Wireless Broadcast Systems Using Distributed Transmission/ Directional Reception (DTDR)," U. S. Patent 5,345,599, September 1994.

[8] J. Adeane, W. Q. Malik and I. J. Wassell, "Error Performance of Ultrawideband Spatial Multiplexing Systems," IET Microwaves, Antennas \& Propagation, Vol. 3, No. 3, March 2009, pp. 363-371.

[9] R. W. Heath Jr. and A. J. Paulraj, "Switching between Diversity and Multiplexing in MIMO Systems," IEEE Transactions on Communications, Vol. 53, No. 6, June 2005, pp. 962-968.

[10] R. W. Heath Jr. and A. J. Paulraj, "Diversity versus Multiplexing in Narrowband MIMO Channels: A Tradeoff Based on Euclidean Distance," IEEE Transactions on Communications, December 2002, pp. 1-26.

[11] R. W. Heath Jr. and A. J. Paulraj, "Characterization of MIMO channels for Spatial Multiplexing Systems," Proceedings of IEEE International Conference on Communications, Helsinki, Vol. 2, 11-14 June 2001, pp. 591-595.

[12] N. Kita, W. Yamada, A. Sato, D. Mori and S. Uwano, "Measurement of Demmel Condition Number for $2 \times 2$ MIMO-OFDM Broadband Channels," Proceedings of IEEE 59th Vehicular Technology Conference, Milan, Vol. 1, 17-19 May 2004, pp. 294-298.

[13] C. Han, A. Doufexi, S. Armour, K. H. Ng and J. McGeehan, "Adaptive MIMO OFDMA for Future Generation Cellular Systems in Realistic Outdoor Environment," Proceedings of IEEE 63rd Vehicular Technology Conference, Melbourne, 7-10 May 2006, pp. 142-146.

[14] C. Han, S. Armour, A. Doufexi, K. H. Ng and J. McGeehan, "Link Adaptation Performance Evaluation for a MIMOOFDM Physical Layer in a Realistic Outdoor Environment," Proceedings of IEEE 64th Vehicular Technology Conference, Montreal, Vol. 74, 25-28 September 2006, pp. 1-5.

[15] B. Muquet, E. Biglieri and H. Sari, "MIMO Link Adaptation in Mobile WiMAX Systems," Proceedings of IEEE Wireless Communications and Networking Conference, Kowloon, 11-15 March 2007, pp. 1810-1813.

[16] A. Forenza, M. R. McKay, I. B. Collings and R. W. Heath Jr., "Switching bewteen OSTBC and Spatial Multiplexing with Linear Receivers in Spatially Correlated
MIMO Channels," Proceedings of IEEE 63rd Vehicular Technology Conference, Melbourne, Vol. 3, 7-10 May 2006, pp. 1387-1391.

[17] A. Forenza, M. R. McKay, A. Pandharipande, R. W. Heath Jr. and I. B. Collings, "Adaptive MIMO Transmission for Exploiting the Capacity of Spatially Correlated Channels," IEEE Transactions on Vehicular Technology, Vol. 56, No. 2, March 2007, pp. 619-630.

[18] C. Wood and W. S. Hodgkiss, "MIMO Channel Models and Performance Metrics," Proceedings of IEEE Global Telecommunications Conference, Washington DC, 26-30 November 2007, pp. 3740-3744.

[19] J. W. Demmel, "The Probability that a Numerical Analysis Problem is Difficult," Mathematics of Computation, Vol. 50, No. 182, 1988, pp. 449-480.

[20] D. Chen, I.-K. Fu, M. Hart and W. C. Wong, "Project IEEE 802.16j Relay Task Group; Channel Models and Performance Metrics for IEEE 802.16j Relay Task Group," IEEE 802.16j MMR Contribution, May 2006.

[21] W. C. Wong, I.-K. Fu, D. Chen, M. Hart and P. Wang, "Comparison of Multipath Channel Models for IEEE 802.16j Relay Task Group," 01-07-2006.

[22] Y. S. Shen and E. Martinez, "WiMAX Channel Estimation: Algorithms and Implementations," Freescale Semiconductor, Application Note, Revision 0, Draft A, July 2007.

[23] M. Hsieh and C. Wei, "Channel Estimation for OFDM Systems Based on Comb-Type Pilot Arrangement in Frequency Selective Fading Channels," IEEE Transactions on Consumer Electronics, Vol. 44, No. 1, 1998, pp. 217-225.

[24] Y. S. Shen and E. Martinez, "Channel Estimation in OFDM Systems," Freescale Semiconductor, Application Note, Revision 0, January 2006.

[25] A. Edelman, "On the Distribution of a Scaled Condition Number," Mathematics of Computation, Vol. 58, No. 197, 1992, pp. 185-190.

[26] T. Tao and Van Vu, "The Condition Number of a Randomly Perturbed Matrix," Proceedings of the 39th Annual ACM Symposium on Theory of Computing, San Diego, 11-13 June 2007, pp. 248-255.

[27] E. K. Hlel, F. Tlili, S. Cherif and M. Siala, "Channel Parameters Estimation for OFDM Systems," International Symposium on Communications, Control and Signal Processing, Marrakech, 13-15 March 2006, pp. 1-4.

[28] X. Y. Tang, M.-S. Alouini and A. J. Goldsmith, "Effect of Channel Estimation Error on M-QAM BER Performance in Rayleigh Fading," IEEE Transactions on Communications, Vol. 47, No. 12, 1999, pp. 1856-1864.

[29] L. Z. Cao and N. C. Beaulieu, "Exact Error-Rate Analysis of Diversity 16-QAM with Channel Estimation Error," IEEE Transactions on Communications, Vol. 52, No. 6, June 2004, pp. 1019-1029.

[30] Y. Mlayeh, F. Rouissi, F. Tlili and A. Ghazel, "Improvement Technique of Channel Estimation in OFDM-MIMO Systems," Proceedings of 16th IEEE International Conference on Electronics, Circuits and Systems, Hammamet, 13-16 December 2009, pp. 467-470. 